\title{
Artículo Original \\ Características y percepción de los servicios madereros en aserraderos de Pucallpa
}

\section{[Features and perception of the timber service industry in Pucallpa]}

\author{
Wagner Pilco-Panduro ${ }^{1}$, Susy Karina Davila-Panduro ${ }^{2}$ y Carlos Antonio Li-Loo Kung ${ }^{3 *}$ \\ ${ }^{1}$ Dirección Regional Sectorial de Agricultura, Departamento de Ucayali, Jr. José Galvez No 287, Pucallpa, Perú. \\ ${ }^{2}$ Facultad de Ciencias de la Educación y Humanidades, Universidad Nacional de la Amazonía Peruana, calle Sgto. Lores \\ $6^{\text {ta }}$ cuadra, Iquitos, Perú. \\ ${ }^{3}$ Facultad de Industrias Alimentarias, Universidad Nacional de la Amazonía Peruana, calle Nauta $5^{\text {ta }}$ cuadra, Iquitos, Perú. \\ *e-mail: carlosli22@hotmail.com
}

\begin{abstract}
Resumen
El objetivo fue determinar las características de la demanda nacional de servicios madereros en la población de la ciudad de Pucallpa durante el año 2011, fue de tipo descriptivo transversal no experimental, la población estuvo conformado por todos los clientes de los aserraderos de la ciudad del Pucallpa que fueron 744 clientes de 4 aserraderos y la muestra fue seleccionada en dos etapas y estuvo conformado por 254 clientes de los cuales 51 fueron del aserradero Cabrera SAC, 87 fueron del aserradero Venao SRLtda, 56 fueron del aserradero Vásquez SRL y 60 fueron del aserradero Pezo EIRL. La técnica que se empleó para la recolección de los datos fue la encuesta, el instrumento fue el cuestionario, para el análisis de los datos se empleó el porcentaje y la media. Los resultados fueron: en las características de los servicios madereros, se observa que el 50,46\% de los aserraderos, están en situación de Regular. En la percepción de la calidad de los servicios madereros el $55,87 \%$ de los aserraderos, están en situación de Regular. En el comportamiento de mercado maderero, el $57,56 \%$ de los aserraderos, de la misma manera. Finalmente en la demanda de los servicios madereros el $54,63 \%$, de los aserraderos, dio el mismo resultado, debiendo los gerentes tomar decisiones correctivas para mejorar y continuar con los comportamientos y procedimiento que demuestren la calidad de la gestión empresarial.
\end{abstract}

Palabras clave: Servicio Maderero, calidad, demanda, gestión.

\begin{abstract}
The aim of this study was to determine the characteristics of the national demand of timber service on the population of Pucallpa City during 2011. This study was transversal- descriptive, non-experimental type. The population was formed by all the clients of sawmills in Pucallpa city, which were approximately 744 clients from 4 different sawmills, the sample was formed by 254 clients taken from Sawmill Cabrera SAC.; 87 were from Sawmill Venao SR Ltda., 56 were taken from Sawmill Vasquez SRL and 60 were from Sawmill Pezo EIRL. The technique used for the collection for the data was the survey and the instrument used for it was the questionnaire. The percentage and media werem used for the analysis of the data. The most relevant results were: about the characteristics of the Timber service industry: it was observed that $50.46 \%$ of sawmills are in situation of Regular. About the perception of the timber service it was observed that $57.56 \%$ of the sawmills are in the situation of Regular. Finally, about the demand of the timber service, $54.63 \%$ of sawmills are in situation of Regular. Having obtained these results the managers need to make corrective decisions in order to improve and continue with the procedures of quality required in the managing of this business.
\end{abstract}

Key words: timber service, quality, demand, management. 


\section{INTRODUCCIÓN}

El sector maderero es un sector rico en la generación de empleo y aprovechamiento adecuado de los recursos naturales. A lo largo de los últimos 50 años sus problemas de gestión, operacionales y de formación se analizaron con cuidado y sus causas identificadas, pero las medidas correctas frecuentemente se convirtieron en intentos truncos y fracasados (Binagorov, 1984).

Lamentablemente muchas de las reformas no han reformado los aspectos más centrales del quehacer empresarial. Los servicios que brindan los aserraderos actualmente no son de alta calidad, están basados en la demanda de muchos clientes y no cuentan con acompañamiento al cliente para dar el mejor uso al producto. Además no están acompañados de un "valor añadido" como posibilidades útiles para su posterior utilización (caso asesoramientos en la compra). Por todo lo mencionado anteriormente, convertir la calidad en la expresión de la satisfacción del cliente de servicios madereros, es uno de los desafíos mayores de quienes promueven este tipo de empresa en la actualidad (Binagorov, 1984).

La satisfacción o insatisfacción del cliente influirá positiva o negativamente en los lazos de lealtad y fidelización que cree con un aserradero. Que esta última logre convencer a un cliente que su servicio es bueno no es tarea fácil (Brown, 1980); de ahí la necesidad de realizar estudios que permitan disponer de las características de las demandas de los servicios madereros en los aserraderos de la ciudad de Pucallpa.

La orientación del mercado hacia los productos con mayor valor agregado genera la oportunidad y a la vez necesidad de diversificación para que los Aserraderos de la ciudad de Pucallpa, incursione en la producción y comercialización de productos maderables terciarios (Bertrand, 1990).

El interés que tienen los aserraderos en procesar maderas, radica en aumentar la competitividad empresarial, ofreciendo a sus clientes un artículo de madera de calidad, que a la vez aporte y enriquezca en habilidades y conocimientos las áreas de aprender, haciendo producción y promoción para la proyección institucional (Egas, 1998).
Existe una oportunidad en el mercado debido a que los grandes aserraderos han enfocado su producción de madera a mercados externos (otros países) y debido a su capacidad productiva se han visto obligados a dejar de vender al mercado interno (Gaither, 2000).

No obstante satisfacer al cliente no es fácil. De un lado, es justo tratar de contentar a quienes hacen posible la existencia de la empresa, pero por otro lado se requiere conocer esa demanda con la finalidad de crear nuevas propuestas y mejorar el funcionamiento de este servicio (Cepal, 1995).

En el presente estudio se pudo obtener la información necesaria para dar a conocer la percepción de los servicios madereros que existen en la ciudad de Pucallpa, de esta manera se identificó las características de la demanda nacional de servicios madereros en la población de la ciudad de Pucallpa durante el año 2011 y cuáles son los aspectos principales para mejorar la calidad de estos servicios y que es lo que demandan los clientes nacionales, por otro lado es importante resaltar que el estudio es el primero en su clase ya que no existen trabajos sobre demanda de servicios madereros en la región.

\section{MATERIALES Y MÉTODOS}

El estudio fue no experimental de tipo descriptivo transversal, porque se pretende describir las características de la demanda de los servicios madereros en un solo momento.

La población del presente estudio estuvo conformada por todos los clientes de los aserraderos de la ciudad de Pucallpa, que son 744 clientes de 40 aserraderos, de los cuales se seleccionaron solo 4 de ellos por la facilidad de recolección de información y porque los demás no desearon estar incluidos en el estudio.

Para determinar la muestra se empleó el muestreo bietápico, en la primera se seleccionaron los aserraderos y luego a los clientes, quedando conformada por 254 clientes, de donde 51 correspondió al aserradero Forestal Cabrera SAC, 87 al aserradero Forestal Venao SRLtda, 56 al aserradero Vásquez SRL y 60 al aserradero Pezo EIRL. 
La técnica que se empleó teniendo en cuenta el tamaño de la muestra fue la encuesta y el instrumento que se empleó en la recolección de la información fue el cuestionario (ver anexo) (Casado, 1997).

\section{RESULTADOS Y DISCUSIÓN}

En el Cuadro 1, se puede observar la opinión acerca de las características de los servicios madereros, en aserraderos de la ciudad de Pucallpa, y se observa que 128 clientes manifestaron el mayor porcentaje de $50,46 \%$ el cual da como resultado una opinión Regular, en los aserraderos Cabrera SAC, Venao SRLtda, Vásquez SRL y Pezo EIRL, esto se debe a que los productos no son de la calidad esperada en su totalidad, (Binagorov, 1984) no son de alta calidad, estos nos demuestran estar fabricados según las especificaciones ofrecidas y en muchos de los casos tampoco están acorde con la necesidad del cliente, esto puede deberse a que los productos están fabricados utilizando técnicas y procedimientos no adecuados para su utilización, pero aún con estos inconvenientes, los productos demuestran estar preparados adecuadamente y tienen un nivel de secado y humedad dentro de lo establecido, así también tienen un buen corte y el tamaño necesario para su utilización, considerando que son producto que medianamente competitivos y a un precio adecuado al mercado nacional, siendo los productos más comercializados: COPAIBA, HUAYRURO y AZUCAR HUAYO, los cuales están dentro del estándar establecido para ventas.

Los servicios madereros en Pucallpa se encuentran en un nivel regular, esto ya lo encontró Binagorov (1984) quien determinó que no son de alta calidad.

En el Cuadro 2, se puede observar el análisis de la opinión acerca de la percepción de la calidad de los servicios madereros, en aserraderos de la ciudad de Pucallpa, y se observa que de 254 clientes, manifestaron el mayor porcentaje de $55,87 \%$ el cual da como resultado una opinión Regular, en los aserraderos Cabrera SAC, Venao SRLtda, Vásquez SRL y Pezo EIRL, esto demuestra que la empresa cuenta con datos técnicos de los productos que comercializa y que en su mayoría cumple con todas las entregas de los productos que comercializa; demuestran estar organizadas y con una adecuada conducción, por otro lado realizan un uso y destino adecuado de los recursos económicos y los gerentes por lo general toman decisiones oportunas y efectivas en la empresa.

En el Cuadro 3, se puede observar el análisis de la opinión acerca del comportamiento de mercado maderero, en aserraderos de la ciudad de Pucallpa, y se observa que de 254 clientes, el mayor porcentaje de 57,56\% el cual da como resultado una opinión Regular, en los aserraderos Cabrera SAC, Venao SRLtda, Vásquez SRL y Pezo EIRL, esto nos da a conocer que el mercado tiene una mediana demanda de los productos, los precios de los productos ofertados no siempre están al alcance de los clientes, hay una buena competencia de otras empresas extranjeras, además de existir productos sustitutos en el mercado. 
Cuadro 1: Opinión acerca de las características de los servicios madereros.

\begin{tabular}{|c|c|c|c|c|c|c|c|c|}
\hline \multirow{2}{*}{$\begin{array}{c}\text { Características de los servicios } \\
\text { madereros }\end{array}$} & \multicolumn{2}{|c|}{ Bueno } & \multicolumn{2}{|c|}{ Regular } & \multicolumn{2}{|c|}{ Deficiente } & \multicolumn{2}{|c|}{ TOTAL } \\
\hline & $\mathbf{N}$ & $\%$ & $\mathbf{N}$ & $\%$ & $\mathbf{N}$ & $\%$ & $\mathbf{N}$ & $\%$ \\
\hline $\begin{array}{l}\text { 1. Los productos son de la calidad } \\
\text { esperada. }\end{array}$ & 136 & $53,54 \%$ & 105 & $41,34 \%$ & 13 & $5,12 \%$ & 254 & $100 \%$ \\
\hline $\begin{array}{l}\text { 2. Los productos demuestran estar } \\
\text { fabricados según las } \\
\text { especificaciones ofrecidas. }\end{array}$ & 107 & $42,13 \%$ & 135 & $53,15 \%$ & 12 & $4,72 \%$ & 254 & $100 \%$ \\
\hline $\begin{array}{l}\text { 3. Los productos están acorde con } \\
\text { la necesidad del cliente. }\end{array}$ & 76 & $29,92 \%$ & 167 & $65,75 \%$ & 11 & $4,33 \%$ & 254 & $100 \%$ \\
\hline $\begin{array}{l}\text { 4. Los productos están fabricados } \\
\text { utilizando técnicas y } \\
\text { procedimientos adecuados para su } \\
\text { utilización. }\end{array}$ & 75 & $29,53 \%$ & 169 & $66,54 \%$ & 10 & $3,94 \%$ & 254 & $100 \%$ \\
\hline $\begin{array}{l}\text { 5. Los productos demuestran estar } \\
\text { preparados con adecuadamente. }\end{array}$ & 57 & $22,44 \%$ & 178 & $70,08 \%$ & 19 & $7,48 \%$ & 254 & $100 \%$ \\
\hline $\begin{array}{l}\text { 6. Los productos presentan un nivel } \\
\text { de secado y humedad dentro de lo } \\
\text { establecido. }\end{array}$ & 44 & $17,32 \%$ & 178 & $70,08 \%$ & 32 & $12,60 \%$ & 254 & $100 \%$ \\
\hline $\begin{array}{l}\text { 7. Los productos muestran un buen } \\
\text { corte. }\end{array}$ & 65 & $25,59 \%$ & 175 & $68,90 \%$ & 14 & $5,51 \%$ & 254 & $100 \%$ \\
\hline $\begin{array}{l}\text { 8. Los productos tiene el tamaño } \\
\text { necesario para su utilización. }\end{array}$ & 68 & $26,77 \%$ & 152 & $59,84 \%$ & 34 & $13,39 \%$ & 254 & $100 \%$ \\
\hline $\begin{array}{l}\text { 9. Los productos tienen un precio } \\
\text { adecuado al mercado nacional. }\end{array}$ & 171 & $67,32 \%$ & 65 & $25,59 \%$ & 18 & $7,09 \%$ & 254 & $100 \%$ \\
\hline $\begin{array}{l}\text { 10. El producto COPAIBA, está } \\
\text { dentro del estándar establecido } \\
\text { para ventas. }\end{array}$ & 184 & $72,44 \%$ & 61 & $24,02 \%$ & 9 & $3,54 \%$ & 254 & $100 \%$ \\
\hline $\begin{array}{l}\text { 11. El producto HUAYRURO, está } \\
\text { dentro del estándar establecido } \\
\text { para ventas. }\end{array}$ & 174 & $68,50 \%$ & 71 & $27,95 \%$ & 9 & $3,54 \%$ & 254 & $100 \%$ \\
\hline $\begin{array}{l}\text { 12. El producto AZUCAR HUAYO, } \\
\text { está dentro del estándar } \\
\text { establecido para ventas. }\end{array}$ & 158 & $62,20 \%$ & 82 & $32,28 \%$ & 14 & $5.51 \%$ & 254 & $100 \%$ \\
\hline Promedio & 110 & $43,14 \%$ & 128 & $50.46 \%$ & 16 & $6.40 \%$ & 254 & $100 \%$ \\
\hline
\end{tabular}


Cuadro 2: Opinión acerca de la percepción de la calidad de los servicios madereros

\begin{tabular}{|c|c|c|c|c|c|c|c|c|}
\hline \multirow{2}{*}{$\begin{array}{l}\text { Percepción de la calidad de los } \\
\text { servicios madereros }\end{array}$} & \multicolumn{2}{|c|}{ Bueno } & \multicolumn{2}{|c|}{ Regular } & \multicolumn{2}{|c|}{ Deficiente } & \multicolumn{2}{|c|}{ TOTAL } \\
\hline & $\mathbf{N}$ & $\%$ & $\mathbf{N}$ & $\%$ & $\mathbf{N}$ & $\%$ & $\mathbf{N}$ & $\%$ \\
\hline $\begin{array}{l}\text { 1. La empresa cuenta con datos técnicos } \\
\text { de los productos que comercializa. }\end{array}$ & 46 & $18,11 \%$ & 159 & $62,60 \%$ & 49 & $19,29 \%$ & 254 & $100 \%$ \\
\hline $\begin{array}{l}\text { 2. La empresa cumple con todas las } \\
\text { entregas de los productos que } \\
\text { comercializa. }\end{array}$ & 56 & $22,05 \%$ & 152 & $59,84 \%$ & 46 & $18,11 \%$ & 254 & $100 \%$ \\
\hline 3. La empresa demuestra estar organizada & 139 & $54,72 \%$ & 102 & $40,16 \%$ & 13 & $5,12 \%$ & 254 & $100 \%$ \\
\hline $\begin{array}{l}\text { 4. La empresa muestra una adecuada } \\
\text { conducción }\end{array}$ & 61 & $24,02 \%$ & 175 & $68,90 \%$ & 18 & $7,09 \%$ & 254 & $100 \%$ \\
\hline $\begin{array}{l}\text { 5. La empresa genera un clima favorable } \\
\text { que facilita las relaciones humanas }\end{array}$ & 26 & $10,24 \%$ & 202 & $79,53 \%$ & 26 & $10,24 \%$ & 254 & $100 \%$ \\
\hline $\begin{array}{l}\text { 6. La empresa realiza un uso y destino } \\
\text { adecuado de los recursos económicos }\end{array}$ & 143 & $56,30 \%$ & 97 & $38,19 \%$ & 14 & $5,51 \%$ & 254 & $100 \%$ \\
\hline $\begin{array}{l}\text { 7. El gerente y el equipo jerárquico toman } \\
\text { decisiones oportunas y efectivas en la } \\
\text { empresa. }\end{array}$ & 161 & $63,39 \%$ & 80 & $31,50 \%$ & 13 & $5,12 \%$ & 254 & $100 \%$ \\
\hline $\begin{array}{l}\text { 8. El gerente y el equipo jerárquico } \\
\text { demuestran conocer las normas } \\
\text { administrativas sobre el personal. }\end{array}$ & 72 & $28,35 \%$ & 174 & $68,50 \%$ & 8 & $3,15 \%$ & 254 & $100 \%$ \\
\hline $\begin{array}{l}\text { 9. El gerente y el equipo jerárquico } \\
\text { demuestran estar capacitado en técnicas } \\
\text { de selección y evaluación de personal }\end{array}$ & 43 & $16,93 \%$ & 199 & $78,35 \%$ & 12 & $4,72 \%$ & 254 & $100 \%$ \\
\hline $\begin{array}{l}\text { 10. El gerente y el equipo jerárquico } \\
\text { demuestran estar capacitado en técnicas } \\
\text { de motivación y relaciones humanas }\end{array}$ & 58 & $22,83 \%$ & 163 & $64,17 \%$ & 33 & $12,99 \%$ & 254 & $100 \%$ \\
\hline $\begin{array}{l}\text { 11. El gerente y el equipo jerárquico } \\
\text { demuestran conocimiento sobre técnicas } \\
\text { de marketing y comercialización, según las } \\
\text { propagandas vistas. }\end{array}$ & 100 & $39,37 \%$ & 132 & $51,97 \%$ & 22 & $8,66 \%$ & 254 & $100 \%$ \\
\hline $\begin{array}{l}\text { 12. El gerente y el equipo jerárquico } \\
\text { demuestran conocimiento del manejo de } \\
\text { control de inventario de la empresa. }\end{array}$ & 168 & $66,14 \%$ & 68 & $26,77 \%$ & 18 & $7,09 \%$ & 254 & $100 \%$ \\
\hline $\begin{array}{l}\text { 13. El gerente y el equipo jerárquico de la } \\
\text { empresa realizan un uso adecuado de la } \\
\text { infraestructura }\end{array}$ & 163 & $64,17 \%$ & 73 & $28,74 \%$ & 18 & $7,09 \%$ & 254 & $100 \%$ \\
\hline $\begin{array}{l}\text { 14. El gerente y el equipo jerárquico } \\
\text { ejecutan adecuadamente los mecanismos } \\
\text { sobre procesos administrativos }\end{array}$ & 81 & $31,89 \%$ & 137 & $53,94 \%$ & 36 & $14,17 \%$ & 254 & $100 \%$ \\
\hline $\begin{array}{l}\text { 15. El gerente y el equipo jerárquico } \\
\text { realizan eficazmente el monitoreo y } \\
\text { supervisión de las actividades productivas. }\end{array}$ & 45 & $17,72 \%$ & 173 & $68,11 \%$ & 36 & $14,17 \%$ & 254 & $100 \%$ \\
\hline Promedio & 89 & $35,20 \%$ & 142 & $55,87 \%$ & 23 & $8,92 \%$ & 254 & $100 \%$ \\
\hline
\end{tabular}


Cuadro 3: Opinión acerca del comportamiento de mercado maderero

\begin{tabular}{|lcccccccc|}
\hline \multicolumn{1}{c}{$\begin{array}{c}\text { Comportamiento de mercado } \\
\text { maderero }\end{array}$} & \multicolumn{2}{c}{ Bueno } & \multicolumn{2}{c}{ Regular } & \multicolumn{2}{c|}{ Deficiente } & \multicolumn{2}{c|}{ TOTAL } \\
\hline $\begin{array}{l}\text { 1. Tamaño de la demanda que } \\
\text { tienen los productos. }\end{array}$ & 182 & $71,65 \%$ & 55 & $21,65 \%$ & 17 & $6,69 \%$ & 254 & $100 \%$ \\
$\begin{array}{l}\text { 2. Precios de los productos } \\
\text { ofertados en el mercado. }\end{array}$ & 38 & $14,96 \%$ & 162 & $63,78 \%$ & 54 & $21,26 \%$ & 254 & $100 \%$ \\
$\begin{array}{l}\text { 3. Nivel de competencia de otras } \\
\text { empresas extranjeras. }\end{array}$ & 22 & $8,66 \%$ & 147 & $57,87 \%$ & 85 & $33,46 \%$ & 254 & $100 \%$ \\
$\begin{array}{l}\text { 4. Concentración de las empresas } \\
\text { maderas en la ciudad. }\end{array}$ & 54 & $21,26 \%$ & 172 & $67,72 \%$ & 28 & $11,02 \%$ & 254 & $100 \%$ \\
$\begin{array}{l}\text { 5. Existencia de productos } \\
\text { sustitutos en el mercado. }\end{array}$ & 9 & $3,54 \%$ & 195 & $76,77 \%$ & 50 & $19,69 \%$ & 254 & $100 \%$ \\
\hline \multicolumn{1}{c}{ Promedio } & $\mathbf{6 1}$ & $\mathbf{2 4 . 0 2 \%}$ & $\mathbf{1 4 6}$ & $\mathbf{5 7 . 5 6 \%}$ & $\mathbf{4 7}$ & $\mathbf{1 8 . 4 3 \%}$ & $\mathbf{2 5 4}$ & $\mathbf{1 0 0 \%}$ \\
\hline
\end{tabular}

En la Figura 1, se puede observar el análisis de la opinión acerca la demanda de los servicios madereros en aserraderos de la ciudad de Pucallpa, y se observa que de 254 clientes, el mayor porcentaje de $54,63 \%$ el cual da como resultado una opinión Regular, en los aserraderos Cabrera SAC, Venao SRLtda, Vásquez SRL y Pezo EIRL, estos resultados permiten indicar que las empresas madereras demuestran estar organizadas, tienen una adecuada conducción, mantienen un clima favorable con los clientes, realizan un uso y destino adecuado de los recursos materiales y recursos económicos. Asimismo los gerentes toman decisiones oportunas y efectivas en las actividades productivas, demuestran conocer las normas administrativas sobre el personal, demuestran estar capacitados en técnicas de selección y evaluación de personal, demuestran estar capacitados en técnicas de motivación y relaciones humanas y finalmente realizan eficazmente el monitoreo y supervisión de las actividades en su empresa.

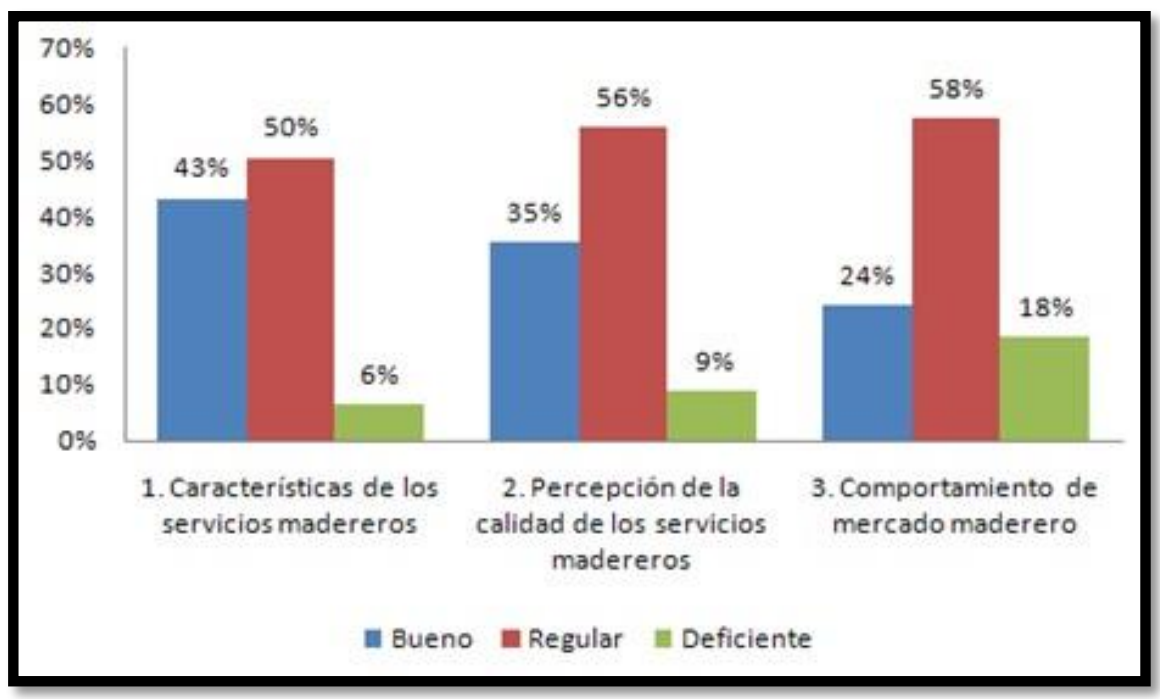

Figura 1: Opinión acerca la demanda de los servicios madereros en la ciudad de Pucallpa 


\section{CONCLUSIÓN}

En el análisis de la Opinión acerca de las características de los servicios madereros, en aserraderos de la ciudad de Pucallpa, se observa que están en situación de Regular, debiendo mejorar las características de los servicios prestados y profundizar los procesos para la mejora de las características del servicio.

Al realizar el análisis de la Opinión acerca de la percepción de la calidad de los servicios madereros, en aserraderos de la ciudad de Pucallpa, se observa que están en situación de Regular, debiendo mejorar la calidad de los servicios madereros y aplicar procesos de control de puntos de críticos para localizar y solucionar problemas en los procesos y así poder elevar la calidad del servicio.

Sobre la Opinión acerca del comportamiento de mercado maderero, en aserraderos de la ciudad de Pucallpa, éstos están en situación de Regular, por lo que se deben mejorar los servicios madereros para que estén a la altura de competir con las otras empresas que existen en la ciudad, así como con los productos sustitutos.

Al realizar el análisis de la Opinión acerca la demanda de los servicios madereros, en aserraderos de la ciudad de Pucallpa, se observa que están en situación de Regular, debiendo los gerentes toman decisiones correctivas para mejorar los procedimiento que demuestren la calidad de la gestión empresarial.

Finalmente se puede concluir que el conocer las características de los servicios madereros, percepción de la calidad y comportamiento de mercado, nos permite enfocar nuestra estrategia de mercado a los mismos, así como poder elegir un efectivo canal publicitario y llegar en forma adecuada y eficaz a cada uno de los potenciales clientes de nuestros servicios.

\section{REFERENCIAS BIBLIOGRAFICAS}

Bertrand L, Prabhakar M. 1990. Control de calidad. Teoría y aplicaciones. Ed. Díaz de Santos S.A. Madrid.

Binagorov GS. 1984. Tecnología del aprovechamiento forestal. Editorial Industria Forestal. Moscu. p.201.

Brown N y Bethel J. 1980. La Industria Maderera. México, LIMUSA-WILEY 397 p.

Casado MM. 1997. Tecnología de las industrias forestales. Tomo I. Serie Forestal 26. Universidad de Valladolid. Escuela Técnica Superior de Ingenierías Agrarias. $191 \mathrm{p}$.

Cepal A. 1995. Estudio sobre la Política de Inversión y de recursos humanos. Montevideo - Argentina $177 \mathrm{p}$.

Denig J. 1990. Control de la calidad en aserraderos de pino del sur. North Carolina Cooperative Extension Service. $47 \mathrm{p}$.

Egas AF. 1998. Consideraciones para elevar los rendimientos en aserraderos con sierras de banda. Tesis para optar por el grado de Doctor en Ciencias Forestales. Universidad de Pinar del Río, Cuba 100 p.

Gaither N. 2000. Administración de producción y operaciones. Editorial Thomsom. 355 p. 
ANEXO

Encuesta realizada en la ciudad de Pucallpa a los clientes de utilizan los servicios madereros.

\begin{tabular}{|c|c|c|c|}
\hline \multirow[t]{2}{*}{ CARACTERÍSTICAS DE LOS SERVICIOS MADEREROS } & \multicolumn{3}{|c|}{ EVALUACIÓN } \\
\hline & BUENO & REGULAR & DEFICIENTE \\
\hline 1. Los productos son de la calidad esperada. & & & \\
\hline $\begin{array}{l}\text { 2. Los productos demuestran estar fabricados según las } \\
\text { especificaciones ofrecidas. }\end{array}$ & & & \\
\hline 3. Los productos están acorde con la necesidad del cliente. & & & \\
\hline $\begin{array}{l}\text { 4. Los productos están fabricados utilizando técnicas y } \\
\text { procedimientos adecuados para su utilización. }\end{array}$ & & & \\
\hline 5. Los productos demuestran estar preparados adecuadamente. & & & \\
\hline $\begin{array}{l}\text { 6. Los productos presentan un nivel de secado y humedad dentro } \\
\text { de lo establecido. }\end{array}$ & & & \\
\hline 7. Los productos muestran un buen corte. & & & \\
\hline 8. Los productos tiene el tamaño necesario para su utilización. & & & \\
\hline 9. Los productos tienen un precio adecuado al mercado nacional. & & & \\
\hline $\begin{array}{l}\text { 10. El producto COPAIBA está dentro del estándar establecido para } \\
\text { ventas. }\end{array}$ & & & \\
\hline $\begin{array}{l}\text { 11.El producto HUAYRURO está dentro del estándar establecido } \\
\text { para ventas. }\end{array}$ & & & \\
\hline $\begin{array}{l}\text { 12.El producto AZUCAR HUAYO está dentro del estándar } \\
\text { establecido para ventas. }\end{array}$ & & & \\
\hline
\end{tabular}

\begin{tabular}{|c|c|c|c|c|}
\hline & \multirow{2}{*}{$\begin{array}{l}\text { PERCEPCIÓN DE LA CALIDAD DE LOS SERVICIOS } \\
\text { MADEREROS }\end{array}$} & \multicolumn{3}{|c|}{ EVALUACIÓN } \\
\hline & & BUENO & REGULAR & DEFICIENTE \\
\hline & $\begin{array}{l}\text { La empresa cuenta con datos técnicos de los productos que } \\
\text { comercializa. }\end{array}$ & & & \\
\hline 2. & $\begin{array}{l}\text { La empresa cumple con todas las entregas de los productos } \\
\text { que comercializa. }\end{array}$ & & & \\
\hline 3. & La empresa demuestra estar organizada & & & \\
\hline 4. & La empresa muestra una adecuada conducción. & & & \\
\hline 5. & $\begin{array}{l}\text { La empresa genera un clima favorable que facilita las } \\
\text { relaciones humanas. }\end{array}$ & & & \\
\hline 6. & $\begin{array}{l}\text { La empresa realiza un uso y destino adecuado de los recursos } \\
\text { económicos }\end{array}$ & & & \\
\hline & $\begin{array}{l}\text { El gerente y el equipo jerárquico toman decisiones oportunas } \\
\text { y efectivas en la empresa. }\end{array}$ & & & \\
\hline 8. & $\begin{array}{l}\text { El gerente y el equipo jerárquico demuestran conocer las } \\
\text { normas administrativas sobre el personal. }\end{array}$ & & & \\
\hline & $\begin{array}{l}\text { El gerente y el equipo jerárquico demuestran estar capacitado } \\
\text { en técnicas de selección y evaluación de personal }\end{array}$ & & & \\
\hline 10. & $\begin{array}{l}\text { El gerente y el equipo jerárquico demuestran estar capacitado } \\
\text { en técnicas de motivación y relaciones humanas }\end{array}$ & & & \\
\hline & $\begin{array}{l}\text { El gerente y el equipo jerárquico demuestran conocimiento } \\
\text { sobre técnicas de marketing y comercialización. }\end{array}$ & & & \\
\hline 12. & $\begin{array}{l}\text { El gerente y el equipo jerárquico demuestran conocimiento del } \\
\text { manejo de control de inventario de la empresa. }\end{array}$ & & & \\
\hline 13. & $\begin{array}{l}\text { El gerente y el equipo jerárquico de la empresa realizan un } \\
\text { uso adecuado de la infraestructura }\end{array}$ & & & \\
\hline 14. & $\begin{array}{l}\text { El gerente y el equipo jerárquico ejecutan adecuadamente los } \\
\text { mecanismos sobre procesos administrativos }\end{array}$ & & & \\
\hline & $\begin{array}{l}\text { El gerente y el equipo jerárquico realizan eficazmente el } \\
\text { monitoreo y supervisión de las actividades productivas. }\end{array}$ & & & \\
\hline
\end{tabular}




\begin{tabular}{|l|l|l|l|}
\hline \multicolumn{2}{|c|}{ COMPORTAMIENTO DE MERCADO MADERERO } & \multicolumn{2}{|c|}{ EVALUACIÓN } \\
\cline { 3 - 4 } & BUENO & REGULAR & DEFICIENTE \\
\hline 1. Tamaño de la demanda que tienen los productos. & & & \\
\hline 2. Precios de los productos ofertados en el mercado. & & & \\
\hline 3. Nivel de competencia de otras empresas extranjeras. & & & \\
\hline 4. Concentración de las empresas maderas en la ciudad. & & & \\
\hline 5. Existencia de productos sustitutos en el mercado. & & & \\
\hline
\end{tabular}

